PROCEEDINGS OF THE

AMERICAN MATHEMATICAL SOCIETY

Volume 132, Number 10, Pages 2997-3005

S 0002-9939(04)07330-7

Article electronically published on June 2, 2004

\title{
A SIMPLE SEPARABLE C*-ALGEBRA NOT ISOMORPHIC TO ITS OPPOSITE ALGEBRA
}

\author{
N. CHRISTOPHER PHILLIPS
}

(Communicated by David R. Larson)

\begin{abstract}
We give an example of a simple separable $\mathrm{C}^{*}$-algebra that is not isomorphic to its opposite algebra. Our example is nonnuclear and stably finite, has real rank zero and stable rank one, and has a unique tracial state. It has trivial $K_{1}$, and its $K_{0}$-group is order isomorphic to a countable subgroup of $\mathbf{R}$.
\end{abstract}

\section{INTRODUCTION}

The purpose of this note is to give an example of a simple separable $\mathrm{C}^{*}$-algebra that is not isomorphic to its opposite algebra. By the opposite algebra $A^{\mathrm{op}}$ of a $\mathrm{C}^{*}$-algebra $A$, we mean the algebra $A$ with the multiplication reversed but all other operations, including the scalar multiplication, the same. (The opposite algebra is isomorphic to the complex conjugate algebra, via the map $x \mapsto x^{*}$.) The existence of type I $\mathrm{C}^{*}$-algebras not isomorphic to their opposites has been known for some time; early examples are due to Raeburn and P. Green, and several examples with additional interesting properties are given in 14]. It has been known for some time that there are von Neumann factors, with separable preduals, of type $\mathrm{II}_{1}[5]$ and type III [4 that are not isomorphic as von Neumann algebras to their opposites. A C*-algebra isomorphism of von Neumann algebras is necessarily a von Neumann algebra isomorphism, by Corollary 5.13 of [17]. So these are simple $\mathrm{C}^{*}$-algebras not isomorphic to their opposite algebras. However, one wants separable examples.

We construct our example by applying a method of Blackadar 22 to the type $\mathrm{II}_{1}$ factor of Corollary 7 of $\left[5\right.$. The resulting $\mathrm{C}^{*}$-algebra is nonnuclear and stably finite, has real rank zero [3] and stable rank one [16], and has a unique tracial state. It has trivial $K_{1}$, and its $K_{0}$-group is order isomorphic to a countable subgroup of $\mathbf{R}$. However, we have little control over other properties. In particular, we cannot specify which subgroups of $\mathbf{R}$ occur, although we can show that there are uncountably many of them. A more natural construction is desirable. See the discussion after Problem 4.5

The recent work on classification of simple nuclear $\mathrm{C}^{*}$-algebras, for example [13] and [10] in the purely infinite case and [7] and [11] in the stably finite case, suggests that all simple nuclear $\mathrm{C}^{*}$-algebras might be isomorphic to their opposites.

Received by the editors July 25, 2002 and, in revised form, February 21, 2003.

2000 Mathematics Subject Classification. Primary 46L35.

Research partially supported by NSF grant DMS 0070776.

(C)2004 American Mathematical Society 
The algebras $A$ and $A^{\text {op }}$ always have the same Elliott invariant, and the nonisomorphism $A \neq A^{\text {op }}$ in our example shows one way in which the Elliott conjecture goes wrong when the nuclearity condition is dropped. Other examples of nonisomorphic simple separable nonnuclear $\mathrm{C}^{*}$-algebras with the same Elliott invariant are known. The algebras have been distinguished by the Haagerup invariant ([15]; proof of Theorem 4.3.8 of [13]), which finite-dimensional operator spaces can be embedded in the algebra (15]; proof of Theorem 4.3 .11 of [13]), quasidiagonality ([8], [15]), approximate divisibility (Theorem 1.4 of [6]; also see Remark 4.3.2 of [13]), and tensor indecomposability of an associated von Neumann algebra (8]). None of these methods is capable of distinguishing a $\mathrm{C}^{*}$-algebra from its opposite algebra.

\section{BLACKADAR'S RESULT AND SOME ANALOGS}

A key ingredient of our construction is the following result of Blackadar, Proposition 2.2 of [2].

Lemma 2.1. Let $N$ be a simple $C^{*}$-algebra, and let $A \subset N$ be a separable $C^{*}$ subalgebra. Then there exists a simple separable $C^{*}$ subalgebra $B$ with $A \subset B \subset N$.

To obtain the other properties necessary for our construction, we need to know that it is possible to find separable intermediate subalgebras preserving other properties from the large algebra. To just prove the existence of a separable simple $\mathrm{C}^{*}$-algebra not isomorphic to its opposite, we only need the next lemma, on traces. The remaining lemmas will be used to show that the algebra can be chosen to have additional good properties. Some are already implicit in previous work.

Lemma 2.2. Let $N$ be a unital $C^{*}$-algebra, and let $A \subset N$ be a separable $C^{*}$ subalgebra. Then there exists a separable $C^{*}$ subalgebra $B$ with $A \subset B \subset N$ such that every tracial state on $B$ is the restriction of a tracial state on $N$.

Proof. For any $\mathrm{C}^{*}$-algebra $D$, let $[D, D]$ denote the linear span of the commutators $[a, b]=a b-b a$ with $a, b \in D$. Also, we let, for any $d \in D$ and $S \subset D$,

$$
\operatorname{dist}(d, S)=\inf \{\|d-x\|: x \in S\} .
$$

Without loss of generality, $A$ contains the identity of $N$. We construct inductively separable $\mathrm{C}^{*}$ subalgebras $B_{n} \subset N$ such that

- $B_{0}=A$;

- $B_{0} \subset B_{1} \subset B_{2} \subset \cdots$;

- for every $b \in B_{n}$, we have $\operatorname{dist}\left(b,\left[B_{n+1}, B_{n+1}\right]\right)=\operatorname{dist}(b,[N, N])$.

We do the induction step; the base case is the same. Given $B_{n}$, choose a countable dense subset $S \subset B_{n}$. For $b \in S$ and $m \in \mathbf{N}$, choose

$l(b, m) \in \mathbf{N} \quad$ and $\quad y_{b, m, 1}, y_{b, m, 2}, \ldots, y_{b, m, l(b, m)}, z_{b, m, 1}, z_{b, m, 2}, \ldots, z_{b, m, l(b, m)} \in N$

such that

$$
\left\|b-\sum_{j=1}^{l(b, m)}\left[y_{b, m, j}, z_{b, m, j}\right]\right\|<\frac{1}{m}+\operatorname{dist}(b,[N, N]) .
$$

Take $B_{n+1}$ to be the separable $\mathrm{C}^{*}$ subalgebra of $N$ generated by $B_{n}$ and the countable set

$$
\left\{y_{b, m, j}, z_{b, m, j}: b \in S, m \in \mathbf{N}, \text { and } 1 \leq j \leq l(b, m)\right\} .
$$


For $c \in B_{n}$ and $\varepsilon>0$, choose $b \in S$ with $\|c-b\|<\frac{1}{3} \varepsilon$, choose $m \in \mathbf{N}$ with $\frac{1}{m}<\frac{1}{3} \varepsilon$, and set

$$
s=\sum_{j=1}^{l(b, m)}\left[y_{b, m, j}, z_{b, m, j}\right] \in\left[B_{n+1}, B_{n+1}\right] .
$$

Then $\operatorname{dist}(c,[N, N])<\frac{1}{3} \varepsilon+\operatorname{dist}(b,[N, N])$, whence

$$
\|c-s\| \leq\|c-b\|+\|b-s\|<\frac{1}{3} \varepsilon+\left[\frac{1}{m}+\operatorname{dist}(b,[N, N])\right]<\varepsilon+\operatorname{dist}(c,[N, N]) .
$$

Since $\varepsilon>0$ is arbitrary, this gives

$$
\operatorname{dist}\left(c,\left[B_{n+1}, B_{n+1}\right]\right) \leq \operatorname{dist}(c,[N, N]),
$$

which completes the induction step.

Now set

$$
B=\overline{\bigcup_{n=0}^{\infty} B_{n}} .
$$

It is clear that for every $b \in \bigcup_{n=0}^{\infty} B_{n}$,

$$
\operatorname{dist}(b,[B, B])=\operatorname{dist}(b,[N, N]),
$$

and equality easily follows for all $b \in B$. It is now immediate that

$$
\operatorname{dist}(b, \overline{[B, B]})=\operatorname{dist}(b, \overline{[N, N]})
$$

for all $b \in B$. This implies that the inclusion of $B$ in $N$ defines an isometric linear map

$$
T: B / \overline{[B, B]} \rightarrow N / \overline{[N, N]} .
$$

Let $\tau: B \rightarrow \mathbf{C}$ be any tracial state. We construct a tracial state $\sigma$ on $N$ such that $\left.\sigma\right|_{B}=\tau$. By continuity and the trace property, $\tau$ induces a linear functional $\bar{\tau}: B / \overline{[B, B]} \rightarrow \mathbf{C}$ with $\|\bar{\tau}\|=1$. The Hahn-Banach Theorem provides a linear functional $\omega: N / \overline{[N, N]} \rightarrow \mathbf{C}$ such that $\omega \circ T=\bar{\tau}$ and $\|\omega\|=1$. Let $\sigma: N \rightarrow \mathbf{C}$ be the composition of $\omega$ with the quotient map $N \rightarrow N / \overline{[N, N]}$. Then $\left.\sigma\right|_{B}=\tau$, and in particular $\sigma(1)=1$. Since $\|\sigma\|=1$, it follows that $\sigma$ is a state. Moreover, $\sigma$ is a trace because it vanishes on $[N, N]$. So $\sigma$ is the required tracial state.

Lemma 2.3. Let $N$ be a unital $C^{*}$-algebra, and let $A \subset N$ be a separable $C^{*}$ subalgebra. Then there exists a separable $C^{*}$ subalgebra $B$ with $A \subset B \subset N$ such that $\operatorname{tsr}(B) \leq \operatorname{tsr}(N)$.

Proof. Without loss of generality, $A$ contains the identity of $N$. Following Definition 1.4 and Proposition 1.6 of [16], we let $r=\operatorname{tsr}(N)$ and we construct $B$ in such a way that the space $\operatorname{Lg}_{r}(B) \subset B^{r}$ (Notation 1.3 of [16]), consisting of all $b=\left(b_{1}, b_{2}, \ldots, b_{r}\right) \in B^{r}$ such that $\left\{b_{1}, b_{2}, \ldots, b_{r}\right\}$ generates $B$ as a left ideal, is dense in $B^{r}$.

We construct inductively separable $\mathrm{C}^{*}$ subalgebras $B_{n} \subset N$ such that

- $B_{0}=A$

- $B_{0} \subset B_{1} \subset B_{2} \subset \cdots$;

- $B_{n}^{r} \subset \overline{\operatorname{Lg}_{r}\left(B_{n+1}\right)}$ for all $n$.

We do the induction step; the base case is the same. Choose a suitable norm on $N^{r}$. Let $S$ be a countable dense subset of $B_{n}^{r}$. For each $b \in S$ and $m \in \mathbf{N}$, use $\operatorname{tsr}(N)=r$ to choose

$$
x_{b, m}=\left(x_{b, m, 1}, x_{b, m, 2}, \ldots, x_{b, m, r}\right) \in \operatorname{Lg}_{r}(N)
$$


such that $\left\|x_{b, m}-b\right\|<1 / m$. By definition, there are

$$
y_{b, m, 1}, y_{b, m, 2}, \ldots, y_{b, m, r} \in N
$$

such that

$$
y_{b, m, 1} x_{b, m, 1}+y_{b, m, 2} x_{b, m, 2}+\cdots+y_{b, m, r} x_{b, m, r}=1 .
$$

Take $B_{n+1}$ to be the separable $\mathrm{C}^{*}$ subalgebra of $N$ generated by $B_{n}$ and the countable set

$$
\left\{x_{b, m, j}, y_{b, m, j}: b \in S, m \in \mathbf{N} \text {, and } 1 \leq j \leq r\right\} .
$$

Clearly each $x_{b, m}$ is in $\operatorname{Lg}_{r}\left(B_{n+1}\right)$, so the induction step is complete.

Now set

$$
B=\overline{\bigcup_{n=0}^{\infty} B_{n}} .
$$

We have

$$
B^{r}=\overline{\bigcup_{n=1}^{\infty} B_{n-1}^{r}} \subset \overline{\bigcup_{n=1}^{\infty} \operatorname{Lg}_{r}\left(B_{n}\right)} \subset \overline{\operatorname{Lg}_{r}(B)},
$$

whence $\operatorname{tsr}(B) \leq r$.

Lemma 2.4. Let $N$ be a $C^{*}$-algebra, and let $A \subset N$ be a separable $C^{*}$ subalgebra. Then there exists a separable $C^{*}$ subalgebra $B$ with $A \subset B \subset N$ such that $\mathrm{RR}(B) \leq$ $\operatorname{RR}(N)$.

Proof. The proof is the same as for Lemma 2.3 Following [3], we simply adjust the indexing and consider only $\operatorname{Lg}_{r+1}(B) \cap\left(B_{\mathrm{sa}}\right)^{r+1}$, etc.

Lemma 2.5. Let $N$ be a $C^{*}$-algebra, and let $A \subset N$ be a separable $C^{*}$ subalgebra. Then there exists a separable $C^{*}$ subalgebra $B$ with $A \subset B \subset N$ such that the map $K_{*}(B) \rightarrow K_{*}(N)$ is injective and induces an order isomorphism of $K_{0}(B)$ with a subgroup of $K_{0}(N)$.

Proof. Unitizing, we may assume that $N$ is unital and that $A$ contains the identity of $N$.

If $D$ is a unital $\mathrm{C}^{*}$-algebra, we write $u \sim_{D} v$ for unitaries $u, v$ in some matrix algebra $M_{m}(D)$ that are homotopic in the unitary group $U\left(M_{m}(D)\right)$. We also write $p \sim_{D} q$ for projections $p, q \in M_{m}(D)$ that are Murray-von Neumann equivalent in $M_{m}(D)$, and $p \precsim_{D} q$ if $p$ is Murray-von Neumann equivalent to a subprojection of $q$ in $M_{m}(D)$. Finally, we write $1_{m}$ for the identity of $M_{m}(D)$.

We construct inductively separable $\mathrm{C}^{*}$ subalgebras $B_{n} \subset N$ such that

- $B_{0}=A$

- $B_{0} \subset B_{1} \subset B_{2} \subset \cdots$;

- for $m \in \mathbf{N}$ and $u \in U\left(M_{m}\left(B_{n}\right)\right)$, if $u \sim_{N} 1_{m}$, then $u \sim_{B_{n+1}} 1_{m}$;

- for $m \in \mathbf{N}$ and projections $p, q \in M_{m}\left(B_{n}\right)$, if $p \sim_{N} q$, then $p \sim_{B_{n+1}} q$;

- for $m \in \mathbf{N}$ and projections $p, q \in M_{m}\left(B_{n}\right)$, if $p \precsim_{N} q$, then $p \precsim_{B_{n+1}} q$.

We do the induction step; the base case is the same. Thus, suppose that $B_{n}$ has been found. Since two unitaries $u$ and $v$ with $\|u-v\|<2$ are homotopic, and since $B_{n}$ is separable, for each $m$ there are only countably many homotopy classes $[u]$ of unitaries $u \in M_{m}\left(B_{n}\right)$. Let $S_{0}$ be the set of all pairs $(m,[u])$ with $m \in \mathbf{N}$ and $u \in U\left(M_{m}\left(B_{n}\right)\right)$ such that $u \sim_{N} 1_{m}$. Then $S_{0}$ is countable. For each $(m,[u]) \in S_{0}$, choose a unitary path $t \mapsto w(t)$ in $M_{m}(N)$ with $w_{0}=u$ and $w_{1}=1$, choose $0=t_{0}<t_{1}<\cdots<t_{k}=1$ such that $\left\|w\left(t_{j}\right)-w\left(t_{j-1}\right)\right\|<2$ for $1 \leq j \leq k$, 
and let $T_{0}^{(m,[u])}$ be the subset of $N$ consisting of all matrix entries of all $w\left(t_{j}\right)$. Then let $T_{0}$ be the countable set

$$
T_{0}=\bigcup_{(m,[u]) \in S_{0}} T_{0}^{(m,[u])} .
$$

It is easy to see from the transitivity of homotopy that whenever $u \in U\left(M_{m}\left(B_{n}\right)\right)$ satisfies $u \sim_{N} 1_{m}$, then also $u \sim_{D} 1_{m}$ for any $\mathrm{C}^{*}$-algebra $D$ containing $B_{n}$ and $T_{0}$.

Since projections $p$ and $q$ with $\|p-q\|<1$ are Murray-von Neumann equivalent, each $M_{m}\left(B_{n}\right)$ contains only countably many Murray-von Neumann equivalence classes of projections. A similar construction produces a countable subset $T_{1} \subset N$ such that whenever $p, q \in M_{m}\left(B_{n}\right)$ are projections that are Murray-von Neumann equivalent in $M_{r}(N)$ but not in $M_{m}\left(B_{n}\right)$, then there exist projections $p_{0}, q_{0} \in$ $M_{m}\left(B_{n}\right)$ and a matrix $s \in M_{m}(N)$ such that $p_{0} \sim_{B_{n}} p$ and $q_{0} \sim_{B_{n}} q$, such that all the entries of $s$ are in $T_{1}$, and such that $s^{*} s=p_{0}$ and $s s^{*}=q_{0}$. It follows that whenever projections $p, q \in M_{m}\left(B_{n}\right)$ satisfy $p \sim_{N} q$, then $p \sim_{D} q$ for any $\mathrm{C}^{*}$ algebra $D$ containing $B_{n}$ and $T_{1}$. By essentially the same method, one can construct a countable subset $T_{2} \subset N$ such that whenever $D$ is a $C^{*}$-algebra containing $B_{n}$ and $T_{0}$, and whenever $p, q \in M_{m}\left(B_{n}\right)$ are projections such that $p \precsim_{N} q$, then $p \precsim_{D} q$. The induction step is now completed by taking $B_{n+1}$ to be the $\mathrm{C}^{*}$ subalgebra of $N$ generated by $B_{n} \cup T_{0} \cup T_{1} \cup T_{2}$.

Now set

$$
B=\overline{\bigcup_{n=0}^{\infty} B_{n}}
$$

For every $m$ and every $u \in U\left(M_{m}(B)\right)$ such that $u \sim_{N} 1_{m}$, there are $n$ and $v \in U\left(M_{m}\left(B_{n}\right)\right)$ such that $v \sim_{B} u$. So $v \sim_{B_{n+1}} 1_{m}$, whence $u \sim_{B} 1_{m}$. This implies that $K_{1}(B) \rightarrow K_{1}(N)$ is injective. By a similar argument, for every $m$ and for any two projections $p, q \in M_{m}\left(B_{n}\right)$ such that $p \sim_{N} q$, we have $p \sim_{B} q$. Therefore $K_{0}(B) \rightarrow K_{0}(N)$ is injective. It remains to prove that $K_{0}(B) \rightarrow K_{0}(N)$ is an order isomorphism onto its image. Since this map preserves order, we need only show that if $\eta \in K_{0}(B)$ is a class whose image in $K_{0}(N)$ is positive, then $\eta>0$. So let $p, q$ be projections in matrix algebras over $B$ such that $\eta=[q]-[p]$, and let $e$ be a projection in some matrix algebra over $N$ such that $[q]-[p]=[e]$ in $K_{0}(N)$. Without loss of generality, there is $n$ such that $p$ and $q$ are in matrix algebras over $B_{n}$. Replacing $p$ and $q$ by $p \oplus 1_{r}$ and $q \oplus 1_{r}$ (which are still in matrix algebras over $B_{n}$ ) for suitable $r$, we may assume that in a suitable matrix algebra $M_{m}(N)$, we can write $q=p_{0}+e_{0}$ with $p_{0} \sim_{N} p$ and $e_{0} \sim_{N} e$. In particular, $p \precsim_{N} q$. By construction $p \precsim_{B_{n+1}} q$, whence $p \precsim_{B} q$. It follows that $\eta=[q]-[p]>0$ in $K_{0}(B)$.

\section{The MAin Result}

Our main result will follow from the following proposition, using a suitable choice of the type $\mathrm{II}_{1}$ factor.

Proposition 3.1. Let $N$ be a type $I I_{1}$ factor with separable predual and with trace $\tau$. Let $G_{0}$ be a countable subgroup of $K_{0}(N)$, which we identify with $\mathbf{R}$ via $\tau_{*}$. Then there exists a simple separable unital weak operator dense $C^{*}$ subalgebra $A \subset N$ such that $\operatorname{tsr}(A)=1, \operatorname{RR}(A)=0, K_{1}(A)=0$, the map $K_{0}(A) \rightarrow K_{0}(N)$ induces an order isomorphism of $K_{0}(A)$ with a subgroup of $K_{0}(N)$ containing $G_{0}$, and $A$ has as unique tracial state the restriction $\left.\tau\right|_{A}$. 
Proof. Let $S \subset N$ be a countable subset that is weak operator dense in $N$. For each $g \in G_{0}$ with $g>0$, choose an integer $m(g)>0$ and a projection $p_{g} \in M_{m(g)}(N)$ such that $\tau\left(p_{g}\right)=g$. Let $P \subset N$ be the unital C* subalgebra of $N$ generated by $S$ and all the matrix entries of all $p_{g}$ for $g \in G_{0} \cap(0, \infty)$. Then $P$ is separable and the image of the map $K_{0}(P) \rightarrow K_{0}(N)$ contains $G_{0}$.

We now construct by induction on $n$ separable subalgebras $A_{n}, B_{n}, C_{n}, D_{n}$, and $E_{n}$ with

$$
P \subset A_{0} \subset B_{0} \subset C_{0} \subset D_{0} \subset E_{0} \subset \cdots \subset A_{n} \subset B_{n} \subset C_{n} \subset D_{n} \subset E_{n} \subset \cdots
$$

and such that, for all $n$,

- $A_{n}$ is simple;

- $B_{n}$ has as unique tracial state the restriction $\left.\tau\right|_{B_{n}}$;

- $\operatorname{tsr}\left(C_{n}\right)=1$;

- $\operatorname{RR}\left(D_{n}\right)=0$;

- the map $K_{0}\left(E_{n}\right) \rightarrow K_{0}(N)$ is an order isomorphism onto its image and the map $K_{1}\left(E_{n}\right) \rightarrow K_{1}(N)$ is injective.

The base case is the same as the induction step, using $P$ in place of $E_{n}$. So we do only the induction step. Suppose the subalgebras have been constructed through $E_{n}$. Use Lemma 2.1 to choose a simple separable $\mathrm{C}^{*}$ subalgebra $A_{n+1}$ with $E_{n} \subset A_{n+1} \subset N$. Use Lemma 2.2 to choose a separable $\mathrm{C}^{*}$ subalgebra $B_{n+1}$ with $A_{n+1} \subset B_{n+1} \subset N$ such that every tracial state on $B_{n+1}$ is the restriction of a tracial state on $N$. The invertible elements in $N$ are dense, since in the polar decomposition $x=s\left(a^{*} a\right)^{1 / 2}$ of any $x \in N$ we can first replace the partial isometry by a unitary and then, with an error of $\varepsilon$, replace $\left(a^{*} a\right)^{1 / 2}$ by $\left(a^{*} a\right)^{1 / 2}+$ $\varepsilon \cdot 1$. So we can use Lemma 2.3 to choose a separable $\mathrm{C}^{*}$ subalgebra $C_{n+1}$ with $B_{n+1} \subset C_{n+1} \subset N$ such that $\operatorname{tsr}\left(C_{n+1}\right)=1$. Use Lemma 2.4 and $\operatorname{RR}(N)=0$ to choose a separable $\mathrm{C}^{*}$ subalgebra $D_{n+1}$ with $C_{n+1} \subset D_{n+1} \subset N$ such that $\mathrm{RR}\left(D_{n+1}\right)=0$. Use Lemma 2.5 to choose a separable $\mathrm{C}^{*}$ subalgebra $E_{n+1}$ with $D_{n+1} \subset E_{n+1} \subset N$ such that the map $K_{1}\left(E_{n+1}\right) \rightarrow K_{1}(N)$ is injective and such that the map $K_{0}\left(E_{n+1}\right) \rightarrow K_{0}(N)$ induces an order isomorphism onto its image.

Now set

$$
A=\overline{\bigcup_{n=0}^{\infty} A_{n}}=\overline{\bigcup_{n=0}^{\infty} B_{n}}=\overline{\bigcup_{n=0}^{\infty} C_{n}}=\overline{\bigcup_{n=0}^{\infty} D_{n}}=\overline{\bigcup_{n=0}^{\infty} E_{n}} .
$$

We verify that $A$ has the required properties. Obviously $A$ is separable. The algebra $A$ is weak operator dense in $N$ because it contains $S$. From $A=\overline{\bigcup_{n=0}^{\infty} A_{n}}$ and simplicity of the $A_{n}$, a standard argument shows that $A$ is simple. Any trace $\tau_{0}$ on $A$ must restrict to a trace on each $B_{n}$, necessarily $\left.\tau\right|_{B_{n}}$. Since $\bigcup_{n=0}^{\infty} B_{n}$ is dense in $A$, it follows that $\tau_{0}=\left.\tau\right|_{A}$. On the other hand, clearly $\left.\tau\right|_{A}$ is a trace on $A$. We have $\operatorname{tsr}(A)=1$ by Theorem 5.1 of [16], because $A=\lim C_{n}$ and $\operatorname{tsr}\left(C_{n}\right)=1$ for all $n$. It is clear that $\operatorname{RR}(A)=0$, because $A=\lim D_{n}$ and $\operatorname{RR}\left(D_{n}\right)=0$ for all $n$. Finally, using the relation $A=\lim E_{n}$, a slightly easier version of the argument of the last paragraph of the proof of Lemma 2.5 shows that the map $K_{1}(A) \rightarrow K_{1}(N)$ is injective and the map $K_{0}(A) \rightarrow K_{0}(N)$ is an order isomorphism onto its image. Since $K_{1}(N)=0$, this immediately gives $K_{1}(A)=0$. Moreover, since $P \subset A$ it follows that $G_{0}$ is contained in the image of $K_{0}(A)$.

Theorem 3.2. Let $G_{0}$ be a countable subgroup of $\mathbf{R}$. Then there exists a simple separable stably finite unital $C^{*}$ subalgebra $A$ with $A \neq A^{\text {op }}$ and such that $A$ has 
stable rank one and real rank zero, $K_{1}(A)=0$, the group $K_{0}(A)$ is isomorphic as a scaled ordered group to a countable subgroup of $\mathbf{R}$ containing $G_{0}$, and $A$ has a unique tracial state.

Proof. Let $N$ be the type $\mathrm{II}_{1}$ factor of Corollary 7 of [5], which is not isomorphic as a von Neumann algebra to $N^{\mathrm{op}}$. Apply Proposition 3.1 with this $N$ and with $G_{0}$ as in the hypotheses, and let $A$ be the resulting $\mathrm{C}^{*}$-algebra. The only property that is not immediate is the nonisomorphism $A \neq A^{\text {op }}$.

Suppose that there is an isomorphism $\varphi: A \rightarrow A^{\mathrm{op}}$. Let $\tau_{0}$ be the unique trace on $A$, and let $\tau_{0}^{\mathrm{op}}$ be $\tau_{0}$ regarded as a trace on $A^{\mathrm{op}}$. Let $\pi$ and $\pi^{\mathrm{op}}$ be the GelfandNaimark-Segal representations of $A$ and $A^{\text {op }}$ associated with $\tau_{0}$ and $\tau_{0}^{\text {op }}$. Then $\tau_{0}=\tau_{0}^{\mathrm{op}} \circ \varphi$ by uniqueness of the traces, whence $\pi$ is unitarily equivalent to $\pi^{\mathrm{op}} \circ \varphi$. It follows that $\pi^{\mathrm{op}}\left(A^{\mathrm{op}}\right)^{\prime \prime}$ is isomorphic as a von Neumann algebra to $\pi(A)^{\prime \prime}$.

We claim that $\pi(A)^{\prime \prime} \cong N$. Let $\tau$ be the trace on $N$, and note that Proposition 3.1 gives $\left.\tau\right|_{A}=\tau_{0}$. We may assume that $N$ is represented in the canonical way on the Hilbert space $L^{2}(N, \tau)$; this is just the Gelfand-Naimark-Segal representation of $N$ associated with $\tau$. All we need to know about it is contained in Proposition III.3.12 of [18]. The Hilbert space $H_{\pi}$ of the representation $\pi$ is by construction a subspace of $L^{2}(N, \tau)$. We show below that the $\mathrm{C}^{*}$-algebra $A$ is dense in $N$ in the norm $\|a\|_{2}=\tau\left(a^{*} a\right)^{1 / 2}$ associated with $L^{2}(N, \tau)$. Since $A$ is contained in $H_{\pi}$, it follows that $H_{\pi}=L^{2}(N, \tau)$. Therefore, using weak operator density of $A$ in $N$, we get $\pi(A)^{\prime \prime}=N$, proving the claim.

To prove the density statement, let $x \in N$. Write $x=a+i b$ with $a, b \in N_{\mathrm{sa}}$. Using the Kaplansky Density Theorem, Theorem 2.3.3 of [12], and the fact that the strong operator topology is metrizable on bounded sets when the predual is separable, find bounded sequences $\left(a_{n}\right)$ and $\left(b_{n}\right)$ in $A_{\mathrm{sa}}$ such that $a_{n} \rightarrow a$ and $b_{n} \rightarrow b$ in the strong operator topology. Set $x_{n}=a_{n}+i b_{n} \in A$. Then $x_{n} \rightarrow x$ and $x_{n}^{*} \rightarrow x^{*}$ in the strong operator topology. Since multiplication is jointly strong operator continuous on bounded sets, it follows that $\left(x_{n}-x\right)^{*}\left(x_{n}-x\right) \rightarrow 0$ in the strong operator topology. The trace $\tau$ is strong operator continuous, so $\left\|x_{n}-x\right\|_{2}^{2}=$ $\tau\left(\left(x_{n}-x\right)^{*}\left(x_{n}-x\right)\right) \rightarrow 0$. This proves density.

Similarly $\pi^{\mathrm{op}}\left(A^{\mathrm{op}}\right)^{\prime \prime} \cong N^{\mathrm{op}}$. But now we have contradicted the property $N \nsucceq$ $N^{\mathrm{op}}$.

\section{Consequences And open problems}

Recall that any real $\mathrm{C}^{*}$-algebra $B$ has a complexification $B_{\mathbf{C}}=B \otimes_{\mathbf{R}} \mathbf{C}$, which is a complex $\mathrm{C}^{*}$-algebra with a conjugate linear automorphism $a \otimes \zeta \mapsto a \otimes \bar{\zeta}$. (We refer to Part II of [9] for the general theory of real $\mathrm{C}^{*}$-algebras.) In particular, $B_{\mathbf{C}}^{\text {op }} \cong B_{\mathbf{C}}$. Therefore we obtain the following corollary.

Corollary 4.1. There exists a simple separable stably finite unital $C^{*}$ subalgebra $A$ that is not isomorphic to the complexification of any real $C^{*}$-algebra.

Remark 4.2. The algebras in Theorem 3.2 are not nuclear, because they are weak operator dense in a factor $N$ that is not hyperfinite. One can certainly force them to be nonexact, and it seems unlikely that the construction can be made to produce exact $\mathrm{C}^{*}$-algebras.

Remark 4.3. Theorem 3.2 produces uncountably many mutually nonisomorphic examples, because no countable union of countable subgroups of $\mathbf{R}$ can contain all countable subgroups of $\mathbf{R}$. 
We close by giving several open problems.

Problem 4.4. Let $F$ be a simple unital AF algebra. Find a simple separable stably finite unital $\mathrm{C}^{*}$ subalgebra $A$ with $A \neq A^{\text {op }}$ and such that $\operatorname{tsr}(A)=1, \operatorname{RR}(A)=0$, and $A$ has the same Elliott invariant as $F$.

As far as we can tell, the methods of [15] will not work, because there is no reason to think the property $A \nsubseteq A^{\text {op }}$ is preserved through the steps of the construction there.

Problem 4.5. Find a more natural example of a simple separable $\mathrm{C}^{*}$-algebra $A$ with $A \neq A^{\text {op }}$.

The obvious approach is to try a $\mathrm{C}^{*}$-algebraic version of the crossed product constructions of $[5]$ or 4 . In [4, type $\mathrm{II}_{\infty}$ factors having no nontrivial central sequences play a key role. An adaptation to $\mathrm{C}^{*}$-algebras will be nontrivial, because the obvious $\mathrm{C}^{*}$-algebraic version of a central sequence behaves rather differently. It follows from Theorem 2.4 of [1] that any separable $\mathrm{C}^{*}$-algebra with no nontrivial central sequences is of type I.

The construction of Corollary 7 of [5] also involves central sequences, although in a different way. Since the factor there, say $N$, is the crossed product of a factor $M$ of type $\mathrm{II}_{1}$ by an action $\alpha$ of $\mathbf{Z} / 3 \mathbf{Z}$, one could hope to find a natural $\alpha$-invariant separable weak operator dense $\mathrm{C}^{*}$ subalgebra $A \subset M$ such that $C^{*}(\mathbf{Z} / 3 \mathbf{Z}, A, \alpha) \subset$ $N$ is simple and has a unique tracial state. This at first seems promising, since $M$ is the tensor product of the hyperfinite type $\mathrm{II}_{1}$ factor and the von Neumann algebra of the free group on four generators. Unfortunately, the action $\alpha$ is obtained from an existence theorem which depends on a cohomology computation.

Problem 4.6. Is there a purely infinite simple separable $\mathrm{C}^{*}$-algebra $A$ such that $A \nRightarrow A^{\mathrm{op}}$ ?

The methods here do not seem to apply to the infinite case. One might hope that a $\mathrm{C}^{*}$-algebraic version of the construction of [4], discussed above, could produce such an example.

Problem 4.7. Is there a separable exact $\mathrm{C}^{*}$-algebra $A$ such that $A \neq A^{\mathrm{op}}$ ?

\section{REFERENCES}

[1] C. A. Akemann and G. K. Pedersen, Central sequences and inner derivations of separable $C^{*}$-algebras, Amer. J. Math. 101 (1979), 1047-1061. MR 80m:46051

[2] B. Blackadar, Weak expectations and nuclear $C^{*}$-algebras, Indiana Univ. Math. J. 27 (1978), 1021-1026. MR 80d:46110

[3] L. G. Brown and G. K. Pedersen, C*-algebras of real rank zero, J. Funct. Anal. 99 (1991), 131-149. MR 92m:46086

[4] A. Connes, A factor not anti-isomorphic to itself, Ann. Math. 101 (1975), 536-554. MR 51:6438

[5] A. Connes, Sur la classification des facteurs de type II, C. R. Acad. Sci. Paris Sér. A 281 (1975), 13-15. MR 51:13706

[6] K. J. Dykema and M. Rørdam, Purely infinite simple $C^{*}$-algebras arising from free product constructions, Canad. J. Math. 50 (1998), 323-341. MR 99d:46074

[7] G. A. Elliott, G. Gong, and L. Li, On the classification of simple inductive limit $C^{*}$-algebras, II: The isomorphism theorem, preprint.

[8] L. Ge and N. C. Phillips, Nonisomorphic simple exact $C^{*}$-algebras with the same Elliott and Haagerup invariants, preprint. 
[9] K. R. Goodearl, Notes on Real and Complex $C^{*}$-Algebras, Shiva Publishing Ltd., Nantwich, 1982. MR 85d:46079

[10] E. Kirchberg, The classification of purely infinite $C^{*}$-algebras using Kasparov's theory, preliminary preprint (3rd draft).

[11] H. Lin, Classification of simple $C^{*}$-algebras with tracial topological rank zero, preprint.

[12] G. K. Pedersen, $C^{*}$-Algebras and their Automorphism Groups, London Mathematical Society Monographs, no. 14, Academic Press, London, New York, San Francisco, 1979. MR 81e:46037

[13] N. C. Phillips, A classification theorem for nuclear purely infinite simple $C^{*}$-algebras, Doc. Math. 5 (2000), 49-114 (electronic). MR 2001d:46086b

[14] N. C. Phillips, Continuous-trace $C^{*}$-algebras not isomorphic to their opposite algebras, International J. Math. 12 (2001), 263-275. MR 2002f:46110

[15] N. C. Phillips, Examples of nonisomorphic nonnuclear simple stably finite $C^{*}$-algebras with the same Elliott invariants, in preparation.

[16] M. A. Rieffel, Dimension and stable rank in the K-theory of $C^{*}$-algebras, Proc. London Math. Soc. Ser. 346 (1983), 301-333. MR 84g:46085

[17] Ş. Strătilă and L. Zsidó, Lectures on von Neumann algebras, translated from the Romanian by S. Teleman, Editura Academiei, Bucharest and Abacus Press, Tunbridge Wells, 1979. MR $81 \mathrm{j}: 46089$

[18] M. Takesaki, Theory of Operator Algebras I, Springer-Verlag, New York, Heidelberg, Berlin, 1979. MR 81e:46038

Department of Mathematics, University of Oregon, Eugene, Oregon 97403-1222

E-mail address: ncp@darkwing.uoregon.edu 